\title{
Desenvolvendo o paradigma da integralidade no cuidado à saúde no PET- Saúde/UEM: relato de experiência: atuação de maneira articulada no cuidado integral à saúde da comunidade
}

Shelyn Akari Yamakami ${ }^{*}$, Adriana Lenita Meyer Albiero ${ }^{* *}$, Mauro Luciano Baesso ${ }^{* * *}$, Mitsue Fujima$\mathrm{ki}^{* * * *}$, Raquel Sano Suga Terada ${ }^{* * * *}$, Renata Corrêa Pascotto ${ }^{* * * *}$

Graduação na Universidade Estadual de Maringá - UEM, Maringá, PR, Brasil. Bolsista PET-Saúde.

** Departamento de Farmácia da Universidade Estadual de Maringá UEM, Maringá, PR, Brasil.

*** Departamento de Física da Universidade Estadual de Maringá UEM, Maringá, PR, Brasil.

Departamento de Odontologia da Universidade Estadual de Maringá - UEM, Maringá, PR, Brasil

\section{RESUMO}

O curso de Odontologia da UEM iniciou sua participação no Projeto PET-Saúde a partir da Portaria Interministerial N. ${ }^{\circ} 3$, de 7 de maio de 2009. Atualmente, o programa conta com cinco grupos tutoriais, incluindo seis cursos do Centro de Ciências da Saúde (CCS) e um do Departamento de Psicologia. O objetivo deste trabalho é relatar a experiência do grupo tutorial vinculado à UBS Mandacaru, em Maringá - PR, que no ano de 2013 trabalhou com o tema "Fortalecimento da Rede Mãe Paranaense no município de Maringá-PR”. A dinâmica de trabalho foi dividida em: Abordagem do tema por meio de oficinas sobre o PETSaúde e a Rede Mãe Paranaense e a realização de palestras e oficinas sobre aleitamento materno. Foi realizada uma pesquisa ajustada à realidade local, além de visitas à UBS e a participação no grupo de apoio às gestantes. A aprendizagem ativa com análise crítica do serviço foi exercida por meio da participação no Conselho Municipal e Local de Saúde. As atividades mostraram que o trabalho individual não supre as necessidades da população, mas sim, um trabalho em equipe. Uma equipe multiprofissional que atue de maneira articulada ao cuidado integral à saúde dos indivíduos. $\mathrm{O}$ PET-Saúde possibilita ao aluno aprender a direcionar as ações baseadas na autonomia e corresponsabilidade, bem como adquirir liderança para levar adiante o compromisso com o bem-estar da população.

Descritores: Aleitamento Materno; Saúde Pública; SUS (Sistema Único De Saúde).

\section{INTRODUÇÃO}

A Universidade Estadual de Maringá foi criada pela Lei Estadual N. ${ }^{\circ} 6.034$, de 06/11/69 (D.O.E. de 10/11/69 p. 1). Atualmente conta com cinco extensões regionais, localizadas nos municípios de Cianorte, 
Diamante do Norte, Goioerê, Cidade Gaúcha, Umuarama e Ivaiporã. Em Maringá, estão matriculados 1.984 alunos, distribuídos nos sete cursos oferecidos no campus sede para formação de profissionais na área da saúde: Odontologia (198), Enfermagem (168), Psicologia (399), Biomedicina (54), Educação Física (609), Farmácia (314) e Medicina (242).

Todos esses cursos tiveram seus projetos pedagógicos reformulados nos últimos anos, em atendimento às diretrizes curriculares nacionais (DCNs) para os cursos de graduação na área da saúde. Por meio da análise dos Projetos Pedagógicos dos Cursos, foi visualizada uma modificação no sentido da inserção de componentes curriculares que privilegiem conteúdos relacionados à Atenção à Saúde, particularmente, à Atenção Básica. Entretanto, segundo alguns autores, a orientação profisssional tem sido muito mais direcionada às intervenções curativas do que à atenção primária. Esses autores complementam que há uma "premente necessidade de reorientação profissional em saúde, tendo em perspectiva as necessidades sociais e os princípios e diretrizes do Sistema Único de Saúde" $" 10$.

Com as experiências nas atividades de ensino, pesquisa e extensão, a UEM tem identificado a imprescindibilidade de ampliar e consolidar sua participação na organização de serviços, na consolidação das redes de atenção básica e fortalecimento da atenção primária. Nesta direção, o Programa Nacional de Reorientação da Formação Profissional em Saúde (Pró-Saúde) e o Programa de Educação pelo Trabalho para a Saúde (PET-Saúde), originados da parceria entre os Ministérios da Saúde e da Educação, mobilizaram muitos atores a elaborarem projetos que busquem a integração ensino/serviço/comunidade para uma for- mação profissional inserida na prática no SUS e cujos resultados levem à reflexão, discussão, avaliação e produção de conhecimento nas universidades, serviços de saúde e comunidade.

Em atendimento à Portaria Interministerial N. ${ }^{\circ} 3$, de 7 de maio de 2009, o curso de Odontologia da UEM iniciou sua participação no Projeto PET-Saúde, e atualmente conta com sete cursos: Odontologia, Enfermagem, Psicologia, Educação Física, Farmácia, Biomedicina e Medicina. O PET-Saúde UEM funciona com cinco grupos tutoriais, vinculados a diferentes UBSs (UBS Mandacaru, UBS Pinheiros, UBS Ney Braga, UBS Requião e UBS Grevíleas) e diversos atores que vivenciam na prática a multiprofissionalidade, a interdisciplinaridade e a integralidade no cuidado à saúde.

O grupo tutorial do PET-Saúde vinculado à UBS Mandacaru propôs ações que visam ao fortalecimento da rede de atenção materno-infantil, em consonância com as diretrizes do Ministério da Saúde e da Secretaria Estadual de Saúde do Paraná, por meio dos Projetos Cegonha (Portaria N. ${ }^{o}$ 1.459, de 24 de junho de 2011) e Rede Mãe Paranaense (Resolução SESA N. ${ }^{\circ}$ 025/2013), com a colaboração mútua dos diferentes setores, profissionais, preceptores e acadêmicos.

Desta maneira, o objetivo deste trabalho foi relatar a experiência vivenciada pelo grupo tutorial do PET-Saúde UEM vinculado à UBS Mandacaru, cujo tema é o "Fortalecimento da Rede Mãe Paranaense no município de Maringá - PR”

\section{MATERIAIS E MÉTODOS Grupo tutorial PET-Saúde UEM UBS Mandacaru}

Este grupo é composto por seis preceptores (quatro da Enfermagem, um da Psicologia e um da Odontologia), vinte 
acadêmicos (doze acadêmicos bolsistas e oito não bolsistas), sob a tutoria de um docente do curso de Odontologia.

\subsection{Cenário de prática}

A rede básica de saúde de Maringá constitui-se de cinco regionais de saúde, que abarcam as vinte e cinco UBSs. Uma destas é a UBS Mandacaru (recebe este nome por situar-se no conjunto habitacional Mandacaru), a qual está localizada na Região Norte da cidade e conta com quatro equipes de Saúde da Família, atendendo $70 \%$ da população de sua área de abrangência. Esta unidade compreende 311 famílias cadastradas no Programa Bolsa Família do Governo Federal e 26 famílias cadastradas no Programa do Leite do Governo Estadual. Entre as atividades desenvolvidas, podemos citar as pesagens mensais e trimestrais das crianças menores de dois anos inseridas nestes programas, para avaliação e acompanhamento do crescimento e desenvolvimento, perfazendo um total de 65 crianças.

O trabalho foi desenvolvido em três cenários de prática: 1) sala de reuniões da Universidade Estadual de Maringá para discussão e compartilhamento de experiências com todos os membros do grupo; 2) UBS Mandacaru (acompanhamento dos atendimentos das gestantes, participação nas reuniões dos grupos de gestantes, pesagem e vacinação das crianças); e 3) Hospital Regional Universitário de Maringá e seu Banco de Leite (acompanhamento das gestantes e do bebê no momento do nascimento até a primeira amamentação e palestras para as gestantes), totalizando oito horas semanais de trabalho.

\subsection{Atividades desenvolvidas}

A proposta deste grupo foi trabalhar com as gestantes dentro da Rede de Atenção à Saúde materno-infantil, também co- nhecida no Paraná como "Rede Mãe Paranaense", desenvolvendo ações voltadas à atenção primária no pré-natal e puerpério e ao acompanhamento do crescimento e desenvolvimento das crianças até os 24 meses de vida.

As atividades podem ser didaticamente divididas em:

1. Sensibilização sobre o tema, com os integrantes do Grupo PET-Saúde, compreendendo:

- Atividade 1: realização de oficina sobre o PET-Saúde e a Rede Mãe Paranaense para revisão de marcos teóricos e metodológicos;

- Atividade 2: realização de palestra com o tema "Fortalecendo a Rede Mãe Paranaense em Maringá";

- Atividade 3: realização de seminários com apresentações sobre temas relacionados à Rede Mãe Paranaense, organizadas pelos acadêmicos;

- Atividade 4: Produção de materiais audiovisuais, folders, manuais dentre outros, a partir da vivência no cenário de prática e voltadas para inovações técnico-científicas e na área de gestão.

2. Desenvolvimento de pesquisa científica, compreendendo:

- Atividade 5: desenvolvimento de pesquisa sobre o funcionamento da Rede Mãe Paranaense com vista a melhorar os indicadores epidemiológicos de saúde da população.

3. Integração ensino-serviçocomunidade, compreendendo:

- Atividade 6: inserção dos alunos, de forma progressiva, nas ações conjuntas com os profissionais da rede;

- Atividade 7: realização de seminários com as gestantes, para discussão de temas visando à conscientização dessas futuras mães; 
- Atividade 8: participação nas reuniões do grupo das gestantes.

4. Análise crítica dos serviços, compreendendo:

- Atividade 9: participação dos alunos e docentes nas reuniões dos conselhos locais e no Conselho Municipal de Saúde de Maringá;

- Atividade 10: discussão e avaliação dos impactos das ações propostas.

5. Educação permanente em saúde, compreendendo:

- Atividade 11: construção teórica coletiva dos temas abordados pelo grupo PET-Saúde, com sua problematização e com reuniões semanais do grupo;

- Atividade 12: participação em eventos científicos, intercâmbio de experiências, participação e apresentação de trabalhos nos seminários do PET- Saúde.

As reuniões para sensibilização sobre o tema foram programadas para expor a todo o grupo o significado do Projeto PETSaúde UEM e a proposta da Rede Mãe Paranaense. Assim os acadêmicos, os preceptores e os docentes puderam ter uma primeira aproximação ao tema. Em um segundo momento, estas reuniões começaram a ser direcionadas ao projeto "Fortalecendo a Rede Mãe Paranaense em Maringá-PR". Palestras sobre o manejo clínico, lactação, fisiologia da mama e o aleitamento materno e outras foram realizadas para os alunos. A partir deste contato, os acadêmicos elaboraram apresentações de seminários, mas sob a óptica de cada área - por exemplo, o aleitamento materno na perspectiva da medicina, da odontologia, da psicologia, da biomedicina, da educação física, da farmácia e da enfermagem. Neste momento, cada curso abordava as ações do profissional da área para o melhor cuidado com as gestantes. Durante este período, os alunos trocaram ideias e discutiram as dificuldades, percepções e sugestões para o melhor desenvolvimento do projeto. Além disso, foram realizadas palestras e seminários sobre assuntos de interesse do grupo, com a utilização de folders, recursos audiovisuais e manuais.

Outra atividade realizada pelo grupo refere-se à produção de pesquisa científica no diagnóstico situacional da população atendida na UBS Mandacaru no que se refere às orientações, assistência e apoio prestados ao incentivo do aleitamento materno exclusivo até os seis meses e continuado com a alimentação complementar oportuna até os dois anos ou mais.

Visitas à UBS foram instituídas semanalmente para que o aluno conhecesse a dinâmica do serviço público e tivesse um maior contato com a população, especialmente as gestantes pertencentes àquela área de abrangência. Nestas visitas foi possível acompanhar os diferentes setores passando pelo acolhimento, sala de enfermagem, vacina, curativos, farmácia, dentre outros. Os acadêmicos abordavam as gestantes na sala de espera e conversavam sobre a importância do aleitamento materno, convidando-as a participarem do grupo das gestantes que acontecia mensalmente.

Este grupo se reunia na terceira quinta-feira do mês na Unidade Básica de Saúde. Nestas reuniões eram feitas palestras para as mães e pais principiantes sobre os cuidados na gestação. Estas palestras tratavam de assuntos como: exercícios físicos para as gestantes, a importância do aleitamento materno, o banho humanizado, mitos e verdades sobre a gestação. Além disso, abria-se um espaço para as mães compartilharem suas dúvidas e inseguranças, bem como suas expectativas em relação à vinda 
do filho. $\mathrm{O}$ evento era encerrado através de uma confraternização com os pais.

Outra atividade de integração com a comunidade era a participação no Conselho Municipal e nos conselhos locais de Saúde. Os alunos e docentes tiveram a oportunidade de acompanhar o processo de eleição dos novos membros dos conselhos, além de conhecer a necessidade local por meio dos moradores e das propostas apresentadas. Todas estas experiências foram compartilhadas e discutidas nas reuniões do grupo e o resultado foi avaliado.

As visitas ao Hospital Regional Universitário de Maringá foram realizadas no setor de Ginecologia e Obstetrícia, para oportunizar ao acadêmico o acompanhamento das puérperas desde o nascimento do bebê e o banho humanizado até a primeira amamentação natural. Muitas orientações foram passadas para as mães - como instruções sobre técnicas de amamentação, a importância do aleitamento materno exclusivo até os seis meses de idade e hábitos deletérios causados pela sucção não nutritiva através de chupetas e mamadeiras. As dúvidas das gestantes foram esclarecidas pelos acadêmicos e pelo preceptor enfermeiro do HUM.

No Hospital os alunos também acompanharam as atividades realizadas no Banco de Leite Humano (BLH) junto com as enfermeiras. Este serviço realiza a coleta, a separação de leite contaminado, faz o teste de acidez, avalia o nível de gordura, realiza o controle de qualidade e armazenamento do leite doado, que atende à demanda dos hospitais com bebês sem suprimento, bebês de baixo peso, imunodeprimidos e em risco de morte que estão na Unidade de Terapia Intensiva.

\section{RESULTADOS}

As reuniões iniciais foram fundamentais para conhecer em maior profundidade a temática do projeto, a proposta e o impacto que este poderia produzir na sociedade. Estes encontros proporcionaram uma melhor interação entre os acadêmicos, os quais compartilharam suas expectativas e dúvidas em relação ao tema. Este momento foi importante para facilitar a comunicação e a organização das atividades do projeto. Todos os membros do grupo participaram ativamente das práticas propostas e contribuíram para as discussões de maneira singular, considerando a diversidade de experiências e opiniões.

As palestras sobre o tema "Fortalecendo a Rede Mãe Paranaense em Maringá" instruíram os alunos sobre a importância do aleitamento materno e a necessidade de conscientização da população. Este conhecimento produziu um novo modo de pensar e impulsionou o grupo a refletir sobre como o trabalho poderia responder às necessidades das gestantes. Cada acadêmico trabalhou o mesmo tema em sua área de atuação e foram realizados seminários para novas discussões.

A troca de conhecimento entre os alunos de diferentes cursos foi essencial para o crescimento individual dos membros do grupo e do grupo como um todo. A clareza de um objetivo comum tornou possível a construção coletiva das propostas para aquela população.

A compreensão da necessidade do aleitamento materno trouxe a reflexão sobre a importância da integralidade dentro da prática profissional, ou seja, o cuidado com a saúde da gestante, trabalhando o acolhi- 
mento, o reconhecimento das necessidades, o respeito à fragilidade de seu momento de vida e as ferramentas para conferir a autonomia no cuidado do bebê e da mãe.

Apesar de o grupo PET-Saúde ter vivenciado um trabalhado em equipe, percebeu-se que dentro da UBS alguns serviços eram fragmentados e dificultados pela interação entre os servidores. Diante desta situação, foi priorizada a interação entre os profissionais e os membros do grupo PETSaúde, no intuito de melhorar a convivência e possibilitar as articulações necessárias. O acadêmico começou a acompanhar as atividades na UBS, conhecendo cada setor para estabelecer um clima favorável e positivo com as equipes multiprofissionais da UBS.

A percepção do aluno em relação às necessidade dos usuários foi um passo necessário para aproximar o aluno à realidade local e para que o grupo pudesse criar propostas de intervenção pertinentes junto à comunidade. $\mathrm{O}$ contato direto com as gestantes permitiu a criação de um espaço de comunicação no qual foi abordada a importância do aleitamento materno aos bebês. Além disso, a participação dos acadêmicos no grupo das gestantes foi também essencial para a criação do vínculo. As gestantes compartilharam suas histórias e mostraram como a gestação era compreendida e sentida por elas, e o aluno teve a oportunidade de absorver as diversas perspectivas da maternidade. $\mathrm{O}$ acadêmico levou para discussão a importância do aleitamento materno, e esta troca de informações foi fundamental no delineamento das ações.

As visitas ao HUM propiciaram um acompanhamento da mãe em ambiente hospitalar nos primeiros momentos de vida do bebê e da criação do vínculo afetivo mãefilho. Também permitiram confrontar as dificuldades do aleitamento materno com sua importância num momento real de muitas expectativas, emoções e aprendizagem.

As visitas ao Banco de Leite Humano mostraram a importância da doação do leite materno, todo o cuidado necessário até a sua disponibilização e a ajuda possibilitada aos bebês nos seus primeiros dias de vida.

No projeto PET-Saúde da UBS Mandacaru, o acadêmico teve a oportunidade de desenvolver um olhar humanizado para o cuidado às gestantes e seus bebês, colocar em prática o trabalho em equipe multiprofissional e interdisciplinar, no intuito de promover a saúde e dar autonomia e responsabilização no cuidado de sua família.

\section{DISCUSSÃO}

Este projeto tem por objetivo fomentar a integração da tríade ensinoserviço-comunidade, por meio de atividades envolvendo a participação dos docentes (tutores), dos profissionais (preceptores) e dos estudantes no desenvolvimento de novas práticas direcionadas à atenção à saúde da população. Esta integração ocorreu em diversos momentos e de diferentes formas, gerando um impacto positivo do Projeto PET-Saúde na formação profissional, na organização do serviço e na reflexão dos profissionais envolvidos no atendimento ao usuário.

O grande número de reuniões realizadas oportunizou a interação entre os acadêmicos, os preceptores e os tutores e serviram para aprimorar a comunicação e a ordenação das atividades. Em um trabalho em equipe ocorrem trocas internas, ou seja, de cada pessoa consigo mesma, e trocas externas, da pessoa com o grupo e o ambiente. Essas trocas são constantes e geram um comportamento que é próprio do desenvol- 
vimento de um grupo. A diversidade biopsicossocial de cada um influenciou positivamente a formação desta equipe PETSaúde, levando-a a um clima de harmonia, o que propiciou a autoaprendizagem e a aprendizagem mútua para um bom planejamento e condução das atividades. O planejamento de ações deve existir e ser participativo, ou seja, todos os membros do grupo precisam envolver-se na elaboração, na aprovação, na execução e na avaliação dessas ações, objetivando o desenvolvimento da tarefa profissional no processo de trabalho ${ }^{15}$.

Além disso, durante a realização das oficinas sobre o tema do projeto "Fortalecendo a Rede Mãe Paranaense em Maringá", foi percebida a importância de cada um dentro do grupo e o potencial de todos juntos, enquanto uma equipe de trabalho. As capacitações da equipe promovem maior qualidade nos serviços prestados e consequentemente aumentam a satisfação dos usuários do SUS; por isso é necessário investir na qualificação do grupo e envolver alunos, professores e profissionais de saúde, com o uso de metodologias ativas e desenvolvidas em pequenos grupos ${ }^{1}$.

O aleitamento materno vai muito além de nutrir a criança, principalmente ao longo do primeiro ano de vida, pois a amamentação é considerada um dos essenciais elementos para crescimento físico, funcional e mental do bebê sendo ainda uma importante forma de diminuir a morbimortalidade infantil ${ }^{11}$. Nesta fase, é inquestionável a importância da alimentação adequada, sendo o aleitamento materno exclusivo (AME) o alimento de escolha para a criança até o $6^{\circ}$ mês de vida, a qual deve continuar a ser amamentada, mas receber também alimentação complementar oportuna, até os dois anos de idade ou mais ${ }^{8}$.
Apesar desta recomendação da Organização Mundial de Saúde (OMS) e do Governo Brasileiro e seus órgãos representativos, a média de tempo de AME no Brasil é de apenas 2,2 meses atingindo um percentual de $41 \%$, segundo a última Pesquisa Nacional de Demografia e Saúde, realizada em 2006 pelo Ministério da Saúde (MS) ${ }^{2,3}$. Assim, práticas alimentares inadequadas adquiridas precocemente estão intimamente relacionadas à morbimortalidade de crianças, representada por doenças infecciosas, afecções respiratórias, cárie dental, desnutrição, excesso de peso e carências específicas de micronutrientes como ferro, zinco e vitamina A. Atualmente, no Brasil, 50\% das crianças menores de dois anos apresentam anemia por deficiência de ferro e $20 \%$ apresentam hipovitaminose $\mathrm{A}$, decorrentes da alimentação inadequada. Estima-se que ações de promoção ao aleitamento materno e à alimentação complementar saudável sejam capazes de diminuir, respectivamente, em até $13 \%$ e $6 \%$, a ocorrência de mortes em crianças menores de cinco anos em todo o mundo ${ }^{4}$.

Neste contexto, a temática escolhida para o Projeto PET-Saúde UEM é muito relevante, tendo em vista a importância do aleitamento materno e da alimentação complementar saudável para o crescimento e desenvolvimento das crianças. Estas ações ajudam na consolidação da Rede Mãe Paranaense (Programa Estadual), em consonância com as diretrizes do Projetos Cegonha (Programa Federal), desenvolvendo ações voltadas à atenção primária no pré-natal e puerpério e ao acompanhamento do crescimento e desenvolvimento das crianças até os 24 meses de vida. Além disso, a integração com os serviços nos diversos níveis de atenção, propondo intervenções junto à comunidade, é um ponto fundamental para 
o sucesso deste projeto.

A atuação em serviços e/ou comunidades desde os estágios iniciais do curso é entendida como uma importante estratégia de reorientação do processo formativo em saúde. A atuação prática de estudantes na Atenção Primária desde o início do curso permite-lhes conhecer uma nova concepção de aprendizagem, na qual se utilizam capacidades prévias e se buscam novos conhecimentos (cognitivos, afetivos e psicomotores) para enfrentar as situações que emergem do cotidiano, construindo assim, maior significado para seu aprendizado e possibilitando a construção de novos saberes ${ }^{7}$. Em acréscimo, o trabalho interdisciplinar permite uma visão ampla e única do processo saúde-doença, pois rompe com a fragmentação disciplinar ${ }^{16}$.

Alguns autores ${ }^{12,13}$ verificaram que a realidade incorporada ao ensino ajuda na formação de profissionais que respondam mais satisfatoriamente às necessidades da população. O avanço substantivo na qualidade dos serviços e das ações de saúde só e possível com a participação efetiva da população, pois esta pode apontar problemas e soluções que atendam mais diretamente às suas demandas ${ }^{14}$. Esse perfil da formação do aluno vem ao encontro do enfoque defendido na integralidade da atenção à saú$\mathrm{de}^{5}$. Além disso, quando estão mais próximos da população, durante as visitas domiciliares da sua área de abrangência, os acadêmicos podem visualizar a realidade das condições de vida das pessoas, com suas diversas limitações (socioeconômicas, geográficas, cognitivas). Isso lhes permite identificar os problemas e as possibilidades de soluções para se atingir a qualidade de vida da população ${ }^{9}$.

O que se vê atualmente nos serviços de saúde é a assistência à gestante oferecida quase exclusivamente vinculada à consulta individual. Assim, o gerar é conduzido pelos profissionais de saúde de modo intervencionista, tornando a assistência e as atividades educativas fragmentadas, sem que a realidade da mulher gestante seja tratada na sua integralidade ${ }^{6}$.Todas as experiências vivenciadas durante o PET-Saúde UEM foram convertidas em conhecimento, auxiliando na consolidação de uma proposta de educação permanente em saúde e provocando reflexão crítica sobre as práticas profissionais, a organização do trabalho, a gestão setorial e o controle social em saúde.

Desta forma, o projeto PET-Saúde tem cumprido o seu papel, estimulando e oportunizando a formação de profissionais com perfil adequado às necessidades do SUS e contribuindo para a melhoria do atendimento à comunidade. A atuação articulada entre os diferentes níveis e setores deve pautar-se pelos princípios da integralidade, visando ao cuidado, ao bem-estar social e à autonomia dos usuários e à manutenção da saúde de toda a população.

\section{CONCLUSÃO}

As experiências vivenciadas no grupo tutorial do PET-Saúde UEM foram fundamentais para a prática de intervenções articuladas no cuidado à saúde da comunidade dentro da Rede Mãe Paranaense. Apesar do grande avanço conquistado por esta equipe, ainda restam muitos desafios a serem enfrentados para um cuidado integral.

$\mathrm{O}$ contato com as gestantes, a troca de informações e a comunicação eficaz foram fundamentais para a compreensão do valor do ser humano, auxiliando na tomada de decisões baseadas na autonomia do indivíduo e em sua corresponsabilização.

Além disso, o desenvolvimento de habilidades de liderança está associado ao compromisso com o bem-estar da população, visando ao aperfeiçoamento das condi- 
ções de trabalho e atendimento para que o SUS se torne mais acolhedor, ágil e resolutivo.

Por fim, o Projeto PET-Saúde UEM tem proporcionado uma formação diferenciada, pela vivência da realidade no SUS e da integração ensino-serviço-comunidade na construção de um sistema mais humanizado, resolutivo e unificado.

\section{REFERENCIAS}

1. Brasil. Ministério da Saúde. Ministério da Educação. Programa Nacional de Reorientação da Formação Profisssional em Saúde - Pró-Saúde: objetivos, implementação e desenvolvimento potencial. Brasília: Ministério da Saúde, 2007.

2. Brasil. Ministério da Saúde. Secretaria de Atenção à Saúde. Departamento de Atenção Básica. Saúde da Criança: nutrição infantil: aleitamento materno e alimentação complementar. Brasília: Ministério da Saúde, 2009-a.

3. Brasil. Ministério da Saúde. Pesquisa Nacional de Demografia e Saúde da Criança e da Mulher: 2006. Brasília, 2009-b.

4. Brasil. Portal da Saúde - SUS. Amamenta e Alimenta Brasil. Disponível em:

http://dab.saude.gov.br/portaldab/ama menta.php . Acesso em 30 de Outubro de 2013-c.

5. Ceccim RB, Feuerwerker LCM. Mudanças na graduação das profissões de saúde sob o eixo da integralidade. Cad Saúde Pública. 2004; 20(5): 14001410.

6. Delfino MRR. O processo de cuidar participante com um grupo de gestantes: repercussões na saúde integral individual coletiva [dissertação]. Tuba- rão: Universidade do Sul de Santa Catarina; 2003.

7. Ferreira RC, Silva RF, Aguer CB. Formação do Profissional Médico: a Aprendizagem na Atenção Básica de Saúde. Ver bras educ med. 2007; 31(1): 52-59.

8. Giugliani ERJ. Amamentação Exclusiva. In: Carvalho MR, Tamez RN. Amamentação - bases científicas. Rio de Janeiro: Guanabara Koogan; 2005. p. $15-25$.

9. Junqueira LP. Novas formas de gestão na saúde: descentralização e intersetorialidade. Saúde e Sociedade. 1997; 6(2): 31-46.

10. Mai LD, Scochi MJ, Garcia BMR et al. A construção de uma práxis interdisciplinar em saúde. Maringá: Eduem, 2011; (Coleção fundamentum 69). p. 54.

11. Pamplona V. Aspectos Psicológicos na Lactação. In: Carvalho MR, Tamez RN. Amamentação - bases científicas. Rio de Janeiro: Guanabara Koogan, 2005; p. 166-176.

12. Pereira JG, Martines WBV, Campinas LLSL, Chueiri PS. Integração Academia, Serviço e Comunidade: um relato de experiência do curso de graduação em medicina na atenção básica no município de São Paulo. O Mundo da Saúde. 2009; 33(1): 99-107.

13. Sanchez HF, Drumont MM, Vilaça EL. Adequação de recursos humanos ao PSF: percepção de formandos de dois modelos de formação acadêmica em odontologia. Ciência \& Saúde Coletiva. $2008 ; 13(2)$ : $523-531$.

14. Terada RSS, Hayacibara MF, Rigolon CJ, Silva MC, Lolli LF, Hidalgo MM. Implementação do Pró-Saúde no Curso de Odontologia da Universidade Esta- 
dual de Maringá. Revista da ABENO. 2010; 10(2): 64-71.

15. Thofehrn MB, Leopardi MT. Teoria dos Vínculos Profissionais: um novo modo de gestão em enfermagem. Rev Texto \& Contexto enferm. 2006; 15(3): 409-17.

16. Vilela EM, Mendes IJM. Interdisciplinaridade e saúde: estudo bibliográfico. Rev Lat-Am Enferm. 2003; 11(4): 525531.

\section{ABSTRACT \\ Developing the paradigm of Comprehen- siveness in health care in PET- \\ Saúde/UEM: experience report: acting in a manner articulated in the integrated community health care}

The course of Dentistry UEM began its participation in Project PET-Saúde from the Ministerial Ordinance No. 3 of May 7, 2009. Today the program has 5 tutorial groups, including 5 courses at the Center for Health Sciences ( CCS ) and 1 Department of Psychology . The aim of this study was to report the experience of the tutorial group linked to UBS Mandacaru in
Maringá - PR, who worked with the theme "Strengthening Mother Network Paranaense in Maringá - PR ". Dynamics of work was divided into : Addressing the issue through workshops on PET - Saúde and Mother Rede Paranaense and lectures and workshops on breastfeeding. The survey was adjusted to local realities, prioritizing the teaching-service integration with UBS visits and participation in support group for pregnant women. The active learning through critical analysis of services, through participation in the Municipal Local Health Board, was also performed. This project has shown that individual work does not meet the needs of the population, but rather a team effort. A multidisciplinary team that acts in coordination in comprehensive health care to individual. The PET-Saúde allows the student to learn how to drive actions based on autonomy and responsibility as well as acquire leadership to carry forward the commitment to the welfare of the population.

Descriptors: Breast feeding. Public Health. SUS (Unified Health System). 